PROCEEDINGS OF THE AMERICAN MATHEMATICAL SOCIETY

Volume 124, Number 7, July 1996

\title{
ESSENTIAL SPECTRUM AND $L_{2}$-SOLUTIONS OF ONE-DIMENSIONAL SCHRÖDINGER OPERATORS
}

\author{
CHRISTIAN REMLING
}

(Communicated by Hal L. Smith)

\begin{abstract}
In 1949, Hartman and Wintner showed that if the eigenvalue equations of a one-dimensional Schrödinger operator possess square integrable solutions, then the essential spectrum is nowhere dense. Furthermore, they conjectured that this statement could be improved and that under this condition the essential spectrum might always be void. This is shown to be false. It is proved that, on the contrary, every closed, nowhere dense set does occur as the essential spectrum of Schrödinger operators which satisfy the condition of existence of $L_{2}$-solutions. The proof of this theorem is based on inverse spectral theory.
\end{abstract}

\section{THE RESUlT}

Let $\tau=-\frac{d^{2}}{d x^{2}}+V(x)$ be a Schrödinger differential expression on $[0, \infty)$. Assume that $\tau$ is regular at 0 and in the limit point case at infinity. Then the self-adjoint realizations $H_{\alpha}$ of $\tau$ in the Hilbert space $L_{2}([0, \infty))$ are given by (see e.g. [1])

$$
\begin{aligned}
D\left(H_{\alpha}\right) & =\left\{u \in L_{2}: u, u^{\prime}\right. \text { absolutely continuous, } \\
& \left.\tau u \in L_{2}, u(0) \cos \alpha+u^{\prime}(0) \sin \alpha=0\right\}, \\
H_{\alpha} u & =\tau u, \quad \alpha \in[0, \pi) .
\end{aligned}
$$

The following result is well-known (compare [1, p. 166]). We recall that the essential spectrum of $H_{\alpha}$ does not depend on $\alpha$. (Of course, when talking about $L_{2}$-solutions of the differential equation $\tau u=\lambda u$ in the sequel, we assume tacitly that $u \neq 0$.)

Theorem 1. If $\tau u=\lambda u$ has no $L_{2}$-solution, then $\lambda$ is in the essential spectrum $\sigma_{\text {ess }}$.

The question whether a converse to Theorem 1 holds has first been investigated by Hartman and Wintner in 1949. They were able to give the following answer [2].

Theorem 2 (Hartman/Wintner). Assume that $\tau u=\lambda u$ has an $L_{2}$-solution for all $\lambda$ in some interval $I=\left(\lambda_{1}, \lambda_{2}\right)$. Then for all $\alpha \in[0, \pi)$ :

a) The point spectrum $\sigma_{p}\left(H_{\alpha}\right)$ is nowhere dense in I, i.e. its closure contains no nonempty open set.

b) There is no continuous spectrum in I.

Received by the editors January 23, 1995.

1991 Mathematics Subject Classification. Primary 34L40; Secondary 47E05, 81Q10.

Key words and phrases. One-dimensional Schrödinger operator, Hartman-Wintner conjecture, $L_{2}$-solution, essential spectrum, inverse spectral theory. 
Hartman and Wintner [2] as well as other authors conjectured that part a) could be improved to: $\sigma_{p}\left(H_{\alpha}\right)$ has no accumulation points in $I$. This is false. In fact, the next theorem does not only disprove this conjecture, but it also shows that Theorem 2a) is sharp!

Theorem 3. Let I be a finite, open interval, and let $S \subset I$ be a closed, nowhere dense set. Then there exists a potential $V(x)$ such that: 1) $\sigma_{\text {ess }} \cap I=S$, 2) $\tau u=\lambda u$ has an $L_{2}$-solution for all $\lambda \in I$.

We would like to point out that $S$ can be uncountable, and this disproves another conjecture.

\section{THE PROOF}

The proof is based on inverse spectral theory. We refer the reader to [3] for a modern treatment. The following lemma is an immediate corollary to the main result of the inverse spectral theory (see [3, Theorem 2.5.1, pp. 41, 42]).

Lemma 1 (Gelfand/Levitan). Let $K \subset \mathbb{R}$ be compact, and let $\mu$ be a finite Borel measure on $K$. Then there exist a potential $V(x)$ and a boundary condition $\alpha$ such that the restriction of the spectral measure $\rho_{\alpha}$ to $K$ coincides with $\mu$.

In the next lemma, we will make use of the Titchmarsh-Weyl $m$-function (consult [4, Chapter 9] for the general theory). We recall that $m_{\alpha}$ has the representation

$$
m_{\alpha}(z)=\cot \alpha+\int_{-\infty}^{\infty} \frac{d \rho_{\alpha}(t)}{t-z} \quad(\operatorname{Im} z>0, \alpha \neq 0) .
$$

A similar formula holds for $\alpha=0$. Using the dominated convergence theorem, we see that

$$
\lim _{\epsilon \rightarrow 0+} \epsilon \operatorname{Im} m_{\alpha}(\lambda+i \epsilon)=\rho_{\alpha}(\{\lambda\})
$$

Furthermore, we have the relationship

$$
m_{\beta}(z)=\frac{m_{\alpha}(z) \cot \gamma-1}{m_{\alpha}(z)+\cot \gamma} \quad(\gamma=\beta-\alpha) .
$$

Lemma 2. The statement of Theorem 3 is equivalent to the existence of sequences $\lambda_{n}, g_{n}$ with the properties: $\lambda_{n} \in I$, the set of accumulation points of $\lambda_{n}$ is $S, g_{n}>0$ and $\sum g_{n}\left(\lambda-\lambda_{n}\right)^{-2}<\infty$ for all $\lambda \in I \backslash\left\{\lambda_{n}: n \in \mathbb{N}\right\}$.

Proof. (i) Let $\lambda_{n}, g_{n}$ be sequences with the above properties. Set $\mu=\sum g_{n} \delta_{\lambda_{n}}$, where $\delta_{x}$ is the Dirac measure, i.e. $\delta_{x}(\{x\})=1, \delta_{x}(\mathbb{R} \backslash\{x\})=0$. Because of Lemma 1 , there is a Schrödinger operator $H_{\alpha}$ realizing the measure $\mu$ as spectral measure. Denote its $m$-function by $m_{\alpha}$, and write according to (1)

$$
m_{\alpha}(z)=\sum \frac{g_{n}}{\lambda_{n}-z}+\int_{\mathbb{R} \backslash I} \frac{d \rho_{\alpha}(t)}{t-z}+\cot \alpha .
$$

Clearly, we have $\sigma_{\text {ess }}\left(H_{\alpha}\right) \cap I=S$. We must show that $\tau u=\lambda u$ has an $L_{2}$-solution for all $\lambda \in I$. By the definition of $\mu$, this is clear if $\lambda \in\left\{\lambda_{n}: n \in \mathbb{N}\right\}$. So let $\lambda \in I \backslash\left\{\lambda_{n}: n \in \mathbb{N}\right\}$. First, we assert that $\lim _{\epsilon \rightarrow 0+} \epsilon^{-1} \operatorname{Im} m_{\alpha}(\lambda+i \epsilon)$ exists and is positive. To see this, it suffices obviously to investigate the first term in (4), which we denote by $m_{\alpha}^{(1)}$

$$
\lim _{\epsilon \rightarrow 0+} \frac{\operatorname{Im} m_{\alpha}^{(1)}(\lambda+i \epsilon)}{\epsilon}=\lim _{\epsilon \rightarrow 0+} \sum \frac{g_{n}}{\left(\lambda-\lambda_{n}\right)^{2}+\epsilon^{2}}=\sum \frac{g_{n}}{\left(\lambda-\lambda_{n}\right)^{2}} .
$$


Here we used the monotone convergence theorem. Furthermore, notice that $\lim _{\epsilon \rightarrow 0+} \operatorname{Re} m_{\alpha}(\lambda+i \epsilon)$ exists, too. Again, we can restrict our attention to $m_{\alpha}^{(1)}$. We have

$$
\sum\left|\frac{g_{n}\left(\lambda-\lambda_{n}\right)}{\left(\lambda-\lambda_{n}\right)^{2}+\epsilon^{2}}\right| \leq \sum \frac{g_{n}}{\left|\lambda-\lambda_{n}\right|} \leq\left(\sum \frac{g_{n}}{\left(\lambda-\lambda_{n}\right)^{2}} \sum g_{n}\right)^{1 / 2}<\infty .
$$

Thus the dominated convergence theorem applies. Estimating the convergence rate yields

$$
\begin{aligned}
\mid \operatorname{Re} & m_{\alpha}(\lambda+i \epsilon)-\lim _{\epsilon \rightarrow 0+} \operatorname{Re} m_{\alpha}(\lambda+i \epsilon) \mid \\
& =\left|\int_{-\infty}^{\infty}\left(\frac{t-\lambda}{(t-\lambda)^{2}+\epsilon^{2}}-\frac{1}{t-\lambda}\right) d \rho_{\alpha}(t)\right| \\
& =\left|\int_{-\infty}^{\infty} \frac{\epsilon^{2}}{(t-\lambda)\left((t-\lambda)^{2}+\epsilon^{2}\right)} d \rho_{\alpha}(t)\right| \\
& \leq \frac{\epsilon}{2} \int_{-\infty}^{\infty} \frac{d \rho_{\alpha}(t)}{(t-\lambda)^{2}}=K \epsilon .
\end{aligned}
$$

The inequality follows from $\epsilon|x| /\left(x^{2}+\epsilon^{2}\right) \leq 1 / 2 \quad \forall x \in \mathbb{R}$. Define $\beta \in[0, \pi)$ by $\lim _{\epsilon \rightarrow 0+} \operatorname{Re} m_{\alpha}(\lambda+i \epsilon)=-\cot (\beta-\alpha)$. Then (2) and (3) lead to

$$
\begin{aligned}
\rho_{\beta}(\{\lambda\}) & =\lim _{\epsilon \rightarrow 0+} \epsilon \operatorname{Im} m_{\beta}(\lambda+i \epsilon) \\
& =\lim _{\epsilon \rightarrow 0+} \frac{\left(1+\cot ^{2}(\beta-\alpha)\right) \epsilon^{-1} \operatorname{Im} m_{\alpha}(\lambda+i \epsilon)}{\left[\epsilon^{-1}\left(\operatorname{Re} m_{\alpha}(\lambda+i \epsilon)+\cot (\beta-\alpha)\right)\right]^{2}+\left[\epsilon^{-1} \operatorname{Im} m_{\alpha}(\lambda+i \epsilon)\right]^{2}}>0,
\end{aligned}
$$

i.e. $\tau u=\lambda u$ has an $L_{2}$-solution, which is the corresponding eigenfunction of $H_{\beta}$.

(ii) We will not use the necessity of the given condition. Moreover, that statement has already been established by Hartman and Wintner, so we refer the reader to [2] for the proof.

Proof of Theorem 3. We construct sequences $\lambda_{n}, g_{n}$ satisfying the conditions of Lemma 2. Since $S$ is closed, we can represent the set $I \backslash S$ as a countable or even finite union $\bigcup J_{n}$ of disjoint, open intervals $J_{n}$. For every $n$, let $x_{k}^{(n)}, y_{k}^{(n)}$ be sequences contained in $J_{n}$ which converge to the left, respectively right, endpoint of $J_{n}$ as $k$ tends to infinity. Take $\left\{\lambda_{n}: n \in \mathbb{N}\right\}=\left\{x_{k}^{(m)}: k, m \in \mathbb{N}\right\} \cup\left\{y_{k}^{(m)}: k, m \in \mathbb{N}\right\}$. Then the set of accumulation points of $\left\{\lambda_{n}\right\}$ is $S$. For if $x \in S, \delta>0$ are given, then, since $S$ is nowhere dense, there is a $y \notin S$ with $|y-x|<\delta$. We have $y \in J_{N}$ for some $N \in \mathbb{N}$, and at least one endpoint $z$ of $J_{N}$ also satisfies $|z-x|<\delta$. Since $z$ is an accumulation point of $\left\{\lambda_{n}\right\}$, there exists a $\lambda_{m}$ with $\left|\lambda_{m}-x\right|<\delta$.

Conversely, assume that $x \notin S$, hence $x \in J_{N}$ for some $N \in \mathbb{N}$. Then the distance between those $\lambda_{n}$ which do not lie in $J_{N}$ and $x$ is greater than the positive distance between $x$ and the nearest endpoint of $J_{N}$. Moreover, by the construction of $\left\{\lambda_{n}\right\}$, the subsequence of the $\lambda_{n_{k}} \in J_{N}$ cannot accumulate at $x$ either, hence $x$ is not an accumulation point of $\left\{\lambda_{n}\right\}$.

Since $S$ is closed, the distance $d(x, S)=\inf _{y \in S}|x-y|$ is positive for all $x \notin S$. Define

$$
\Lambda_{m}=\left\{\lambda_{n}:|I| 2^{-m}<d\left(\lambda_{n}, S\right) \leq|I| 2^{-m+1}\right\} \quad(m \in \mathbb{N}),
$$

where $|I|$ is the length of the basic interval $I$. Then the sets $\Lambda_{m}$ are disjoint, and their union is the whole set $\left\{\lambda_{n}: n \in \mathbb{N}\right\}$. We rearrange the $\lambda_{n}$ by writing 
$\Lambda_{m}=\left\{\lambda_{m 1}, \lambda_{m 2}, \ldots\right\}$, and we take $g_{m n}=2^{-3 m-n}$. If $\lambda \in S$, then

$$
\sum_{m, n} \frac{g_{m n}}{\left(\lambda-\lambda_{m n}\right)^{2}} \leq \sum_{m, n} 2^{-3 m-n} 2^{2 m}|I|^{-2}=|I|^{-2} \sum_{m, n} 2^{-m-n} \leq|I|^{-2}<\infty,
$$

and if $\lambda \notin S \cup\left\{\lambda_{m n}\right\}$, then there is a $d>0$ with $\left|\lambda-\lambda_{m n}\right| \geq d$ for all $m, n$, hence also in this case $\sum g_{m n}\left(\lambda-\lambda_{m n}\right)^{-2} \leq d^{-2} \sum g_{m n}<\infty$.

\section{REFERENCES}

1. J. Weidmann, Spectral theory of ordinary differential operators, Lecture Notes in Math., vol. 1258, Springer-Verlag, Berlin and Heidelberg, 1987. MR 89b:47070

2. P. Hartman and A. Wintner, A seperation theorem for continuous spectra, Amer. J. Math. 71 (1949), 650-662. MR 11:109i

3. B. M. Levitan, Inverse Sturm-Liouville problems, VNU Science Press BV, Utrecht, 1987. MR 89b:34001

4. E. A. Coddington and N. Levinson, Theory of ordinary differential equations, McGraw-Hill, New York, 1955. MR 16:1022b

Universität Osnabrück, Fachbereich Mathematik/Informatik, Albrechtstr. 28, D49069 OsnabrüCK, Germany

E-mail address: cremling@chryseis.mathematik.uni-osnabrueck.de 